\title{
An Overview of Array Signal Processing and Beam Forming Techniques
}

\author{
Vaibhav \\ S.N.University \\ Rachi, India
}

\author{
Shiv Sunder Lokesh \\ ECE Department \\ Gyan Vihar University \\ Jaipur, India
}

\author{
Abinet Tesfaye \\ Electrical Engineering \\ MeU,Africa \\ Illu Babora, Africa
}

Tasew Ababe

Electrical Engineering

MeU,Africa

Illu Babora, Africa

\begin{abstract}
For use as hydrophones, projectors and underwater microphones, there is always a need for calibrated sensors. Overview of multi path and effect of reflection on acoustic sound signals due to various objects is required prior to finding applications for different materials as sonar domes, etc. There is also a need to overview multi sensor array processing for many applications like finding direction of arrival and beam forming. Real time data acquisition is also a must for such applications.
\end{abstract}

Keywords: Array signal processing; Uniform linear array; Beam Forming; Delay and Sum Beam Former; Spatial FFT.

\section{INTRODUCTION}

Array signal processing is a part of signal processing that uses sensors that are organized in patterns, or arrays, to detect signals and to determine information about them as shown in Fig.1. One of the applications of array signal processing involves detecting acoustical signals. The sensor in this overview is Hydrophones.

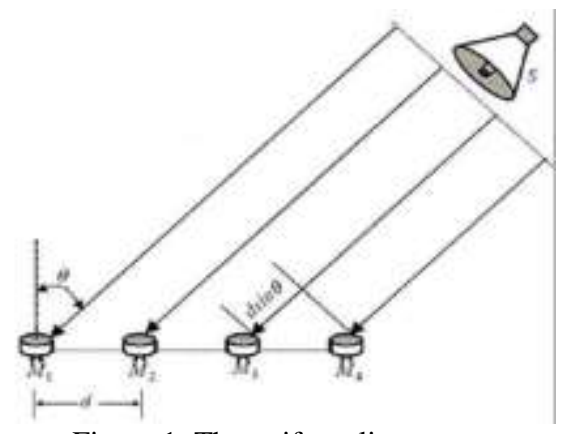

Figure 1: The uniform linear array

\section{NEED}

The goal of this overview is to develop an array which can listen from a specific direction, while attenuating signals not from the direction of interest. A six element uniform linear array can be created in order to determine the direction of the source of specific frequency signals. Because the uniform linear array is one dimensional, there is a surface of ambiguity on which it is unable to determine information about signals. For example, it suffers from "front-back ambiguity", meaning that signals incident from" mirror location" at equal angles on the front and back sides of the array undistinguishable. Without a second dimension, the uniform linear array is also unable to determine how far away a signal's source is or how high above or below the array's level the source is.

\section{UNIFORM LINEAR ARRAY (ULA)}

When constructing any array, the design specification should be determined by the properties of the signals that the array will detect. All acoustic waves travel at the speed $1500 \mathrm{~m} / \mathrm{s}$ in underwater channel [6-8]. The physical relationship describing acoustic waves is similar to that of light $\lambda \mathrm{f}=\mathrm{c}$. The frequencies of signals that an array detects are important because they determine constraints on the spacing of the sensors. The array's sensors sample incident signal in space and, just as aliasing occur in analog to digital conversion when the sampling rate does not meet the Nyquist criterion, aliasing can also happen in space if the sensors are not sufficiently close together[3]. A useful property of the uniform linear array is that the delay from one sensor to the next is uniform across the array because of their equidistant spacing. Trigonometry reveals that the additional distance the incident signal travels between sensors is $\mathrm{d} \operatorname{Sin}(\theta) /$ Thus, the time delay between consecutive sensors is give

$$
\zeta=\mathrm{d} \operatorname{Sin}(\theta) / \mathrm{c}
$$

Say the highest narrowband frequency we are interested is $\mathrm{f}_{\max }$. To avoid spatial aliasing, we would like to limit phase difference between spatially sampled signals to $\pi$ or less because phase difference above $\pi$ cause incorrect time delays to be seen between received signals. Thus, we give the following condition:

$2 \pi \mathrm{f}_{\max } \zeta \leq \pi$

Substituting for $\zeta$ from equation (1) into (2)

$2 \pi \mathrm{f}_{\max } \mathrm{d} \operatorname{Sin}(\theta) / \mathrm{c} \leq \pi$ 
The maximum delay according to equation 1 occurs for $\theta$ $=90^{\circ}$, so we obtain fundamentally important condition from equation (4)

$\mathrm{d} \leq \mathrm{c} / 2 \mathrm{fmax}$

For $40 \mathrm{KHz}$ frequency spacing should be less than $1.87 \mathrm{~cm}$ according to equation (5). The direction perpendicular to the length of the array is taken as broadside of the array. All angles to the right or clockwise from the broadside are considered positive by convention up to $+90^{\circ}$. All angles to the left or counter clockwise from the broadside are considered negative up to $-90^{\circ}$.

\subsection{Ambiguity of the ULA}

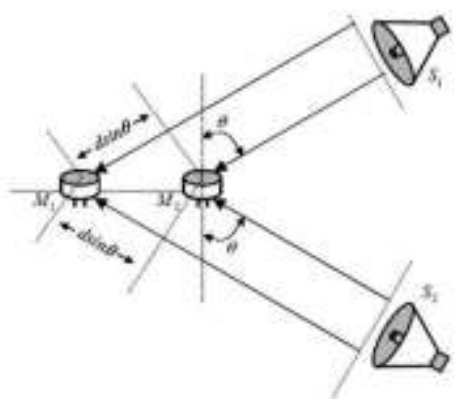

Figure 2 The ULA is unable to distinguish signals from its front or back side, or signals above or below it.

The limitation of the uniform linear array as shown in figure 2 create problem for locating acoustic source with much accuracy [4]. The array's design is highly extensible, however, and it is an important building block for more

\section{BEAM FORMING}

Beam forming is the process of trying to concentrate the array to sounds coming from only one particular direction. Spatially, this would look like a large double shaped lobby aimed in the direction of interest [5]. Making a beam former is crucial to meet one of the goals of the study which is to listen to sound in one direction and The best way to not listen noisy directions is to just steer all your energy towards listening in one direction. This is an important concept because it is not just used for array signal processing; it is also used in many sonar systems as well [1].

\subsection{Delay and Sum Beam Former}

It is good first step to implement delay and sum beam former, because it is the simplest example. The delay and sum beam former is based on the idea that if a uniform linear array is being used, then the output of each sensor will be the same, except that each one will be delayed by a different amount. So, if the output of each sensor is delayed appropriately then we add all the outputs together the signal that was For $k=f n$, and zero otherwise. Here normalized DFT is used.

Now the $\mathrm{N}$ spectra from each of the array's sensors according to equation (10) is calculated, to find how certain frequencies (of interest $\mathrm{f}_{\mathrm{o}}$ from different angles with respect to the array's broadside) are distributed [7]. This DFT is performed by taking frequency component from each received signal that corresponds to $f_{o}$ and concatenating them into an array. Then array is zero padded to make a length that is power of two in order to calculate the FFT. This FFT gives a spectrum that is digital sinc function centered at $f_{o} \zeta$. The value of the sinc represents the magnitude of the frequency $\mathrm{f}_{\mathrm{o}}$ at an angle theta. complex arrays such as cube, which uses multiple linear arrays or more exotic shapes such as a circle [1].

\subsection{Spatial FFT Approach of Beam Forming}

Let us consider the narrow band signals of the form

$\mathrm{X}(\mathrm{t})=\exp (\mathrm{j} 2 \pi \mathrm{ft})$

Where, $\mathrm{f}$ is the frequency of the signal. If we have $\mathrm{N}$ sensors numbered from $n=0,1 \ldots \ldots . . . \mathrm{N}-1$, Then the delayed version of $x(t)$ that arrive at each hydrophone $n$ are

$\mathrm{Xn}(\mathrm{t})=\exp (\mathrm{j} 2 \pi \mathrm{f}(\mathrm{t}-\mathrm{n} \zeta)$

Thus, the first sensor $\mathrm{n}=0$ has zero delay, while the signal arrives at the second sensor $n=1$ one unit delay later then at the first, and so on for each sensor. Then, the signal is sampled at each hydrophones in the process of ADC, and let the sampled signal $\mathrm{Xn}(\zeta)=\mathrm{Xn}(\mathrm{mT})$, where $\mathrm{m}$ is the integer. This gives us the sampled sinusoids at each sensor

$\mathrm{Xn}(\mathrm{r})=\exp (\mathrm{j} 2 \pi \mathrm{fr}-\mathrm{n} \zeta)$

Even though each sensor receives the same frequency signal, recall that delays $\mathrm{X}(\mathrm{t}-\mathrm{n} \zeta)$ in time correspond to modulation by $\exp (-\mathrm{jn} \zeta)$ in frequency, so the spectra of the received signals at each sensor are not identical. The first discrete Fourier transform looks at the temporal frequency content at each sensor

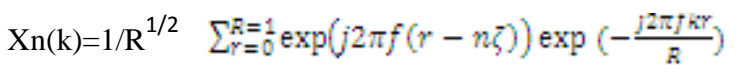

$X n(k)=\exp (-j 2 \pi f n \zeta) / R^{1 / 2}$

Because of the shape of the lobes of the sinc, which look like beams at the various angles the process of using the array to look for signals in different directions is called beam forming. This technique is used frequently in array processing and enables detection of the direction of arrival of certain frequencies [2].

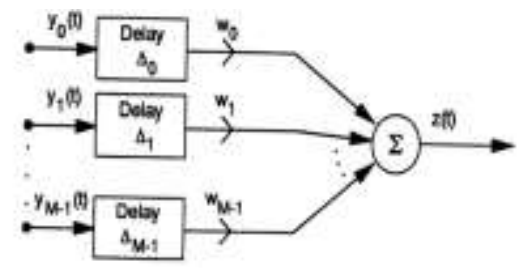

Figure 3 Sum and delay beam former

\section{CONCLUSION}

Basic theory of linear array is studied and constraints are derived that are useful for implementation of algorithms. The important condition for spacing between sensors of uniform linear array to avoid aliasing is derived in this overview.

\section{REFERENCES}

[1] C.H. Cheng, "Signal Processing for Optical Communication,” IEEE Signal Processing Magazine, 
vol. 23, no. 1, pp. 88-96, 2006.

[2] G. Keiser, "Optical Fiber Communication," McGrawHill International series, Third Edition, 2000.

[3] C.D. Poole, J.M. Wiesenfeld, D.J. DiGiovanni, A.M Vengsarkar, "Optical fiber-based dispersion compensation using higher order modes near cutoff," Journal of Lightwave Technology, vol. 12, no. 10, pp. 1746-1758, 1994.

[4] N.M. litchinitser, D.B. Patterson, "Analysis of Fiber Bragg Gratings for Dispersion Compensation in Reflective and Transmissive Geometries," Journal of Lightwave Technology, vol. 15, no. 8, pp. 1303-1313, 1997.

[5] D. Pastor, J. Capmany, D. Ortega, V. Tatay, and J. Marti, "Design of Apodized Linearly Chirped Fiber Gratings for Dispersion Compensation," Journal of Lightwave Technology, vol. 14, no. 11, pp. 25812588, 1996

[6] P.M. Watts, V. Mikhailov, S. Savory, P. Bayvel, M. Glick, M. Lobel, B. Christinsin, P. Krikpatrick, S. Shange, and R.I. Killey, "Performance of Single-Mode Fibers Links using Electronic Feed-Forward and Decision Feedback Equalizers," IEEE Photonics Technology Letter, vol. 17, no. 10, pp. 2206-2208,2005

[7] S. Cao, J. Chen, J.N. Damask, C.R. Doerr, L. Guiziou, G. Harvey, Y. Hibino, H.Li, S. Suzuki, K.Y. Wu, and P. Xie, "Interleaver Technology: Comparisons and Applications Requirements," Journal of Lightwave Technology, vol. 22, no. 1, pp. 281-289, 2004.

[8] S.K. Mitra, K. Hirano, "Digital All Pass Filters," IEEE transaction circuits and systems, vol. CAS 21, no. 9, pp. 688-700, 1974. 